\title{
Enrolling children with acute lymphoblastic leukaemia on a clinical trial improves event- free survival: a population-based study
}

Caron Strahlendorf ${ }^{1,9}$, Jason D Pole ${ }^{2,9}$, Randy Barber ${ }^{3}$, David Dix ${ }^{1}$, Ketan Kulkarni ${ }^{4}$, Emilie Martineau ${ }^{5}$, Alicia Randall ${ }^{4}$, David Stammers ${ }^{6}$, Douglas Strother ${ }^{7}$, Tony H Truong $^{7}$ and Lillian Sung ${ }^{\star}, 8$

${ }^{1}$ British Columbia Children's Hospital, 4480 Oak St, Vancouver, BC V6H 3N1, Canada; ${ }^{2}$ Paediatric Oncology Group of Ontario, 480 University Avenue, Suite 1014, Toronto, ON M5G 1V2, Canada; ${ }^{3}$ C17 Council, 11405 - 87 Avenue, Edmonton, AB T6G 1C9, Canada; ${ }^{4}$ WWK Health Centre, 5980 University Ave, Halifax, NS B3K 6R8, Canada; ${ }^{5}$ Centre Hospitalier Universitaire de QuebecUniversite Laval, 2705, Boulevard Laurier, Quebec City, QC G1V 4G2, Canada; ${ }^{\circ}$ Royal University Hospital, 103 Hospital Dr, Saskatoon, SK S7N OW8, Canada; ${ }^{7}$ Alberta Children's Hospital, 2888 Shaganappi Trail NW, Calgary, AB T3B 6A8, Canada and ${ }^{8}$ The Hospital for Sick Children, 555 University Avenue, Toronto, ON M5G 1X8, Canada

Background: The objectives of this study were to describe the impact of trial enrollment at diagnosis on event-free and overall survival in paediatric acute lymphoblastic leukaemic (ALL) using a population-based approach.

Methods: We conducted a retrospective cohort study that included children newly diagnosed with ALL between 1 and 14 years of age. The data source was the Cancer in Young People in Canada (CYP-C) national paediatric cancer population-based database. We conducted univariate and multiple Cox proportional hazards models.

Results: There were 2569 children with ALL; 1408 (54.8\%) were enrolled on a clinical trial at initial diagnosis. Event-free survival at 5 years was $89.8 \% \pm 0.9$ vs $84.1 \% \pm 1.2$. ( $(P<0.0001)$ for those enrolled and not enrolled on a clinical trial, respectively. Overall survival at 5 years was higher for those enrolled $(94.1 \% \pm 0.7)$ vs not enrolled $(90.5 \% \pm 1.0 ; P=0.001)$. In a model that adjusted for demographic, leukaemic and socioeconomic factors, enrollment on trials was significantly associated with better event-free survival (hazard ratio (HR) 0.67, 95\% confidence interval (Cl) $0.47-0.95 ; P=0.023$ ), but not overall survival ( $\mathrm{HR} 0.69,95 \% \mathrm{Cl} 0.44-1.08 ; P=0.102$ ).

Conclusions: Event-free survival was significantly better in children with ALL enrolled on a clinical trial. Future research should identify barriers to clinical trial enrollment for children with ALL.

There has been debate about the impact of enrollment on clinical trials and survival outcomes for children with cancer. Retrospective studies found that patients enrolled on clinical trials have better outcomes compared with patients not enrolled on clinical trials (Stiller and Eatock, 1994; Wagner et al, 1995). However, a systematic review determined that these studies were limited because of confounding and selection bias, and thus concluded that the impact of trial enrollment is not clear (Peppercorn et al, 2004).
Further, a Cochrane review found that, in general, outcomes are similar between those who do and those who do not participate in randomised trials (Vist et al, 2008).

We recently used the Cancer in Young People in Canada (CYPC) database, a national paediatric cancer population-based data source, to describe the proportion of all children with cancer enrolled on a clinical trial and to describe factors associated with enrollment. About one in four children with cancer were enrolled

*Correspondence: Dr L Sung; E-mail: lillian.sung@sickkids.ca

${ }^{9}$ Co-first authors.

Received 25 July 2017; revised 22 November 2017; accepted 23 November 2017; published online 30 January 2018

(C) 2018 Cancer Research UK. All rights reserved 0007-0920/18 
on a clinical trial (Pole et al, 2017). The most common reasons cited for non-enrollment were lack of an available trial and physician choice. Acute lymphoblastic leukaemic (ALL) is the most common paediatric cancer, accounting for approximately onethird of all cancers in children aged 0-14 years of age. We found that children with ALL had the highest rates of enrollment among paediatric cancer diagnoses (Pole et al, 2017).

Given this data, we next wanted to determine if enrollment on a clinical trial is associated with better survival for children with ALL. Therefore, the objectives of this analysis were to describe the impact of trial enrollment at diagnosis on event-free survival (EFS) and overall survival (OS) in paediatric ALL.

\section{MATERIALS AND METHODS}

Population of interest and sampling methods. We included children with newly diagnosed ALL with ICD-O M codes 9835, 9836 and 9837; these codes define patients with precursor lymphoblastic leukaemic NOS, B-cell and T-cell, respectively. Other inclusion criteria were 1-14 years of age, diagnosed between 01 January 2001 and 31 December 2012, and treated at one of the 17 paediatric oncology centres in Canada. We excluded patients in whom enrollment status was unknown, those diagnosed $<1$ year of age (infant ALL) and those with Burkitt's leukaemic. We chose to exclude infant and Burkitt's ALL as the treatment approach is fundamentally different compared with precursor lymphoblastic leukaemic.

Data source. We used the data from CYP-C, a population-based registry that aims to include all paediatric patients with cancer diagnosed between 0 and 14 years of age since 2001, who were diagnosed and treated at one of the 17 tertiary paediatric oncology centres in Canada. Two data collection approaches are used for CYP-C data. For the 5 centres in Ontario, the data are transferred to CYP-C from the Paediatric Oncology Group of Ontario (POGO) Networked Information System (POGONIS), which is a provincial population-based registry that includes similar data to CYP-C. For the 12 centres outside of Ontario, the data are entered directly into CYP-C. Elements captured by both databases include the following: (1) demographic variables, including sex, date of birth, postal code and race; (2) diagnostic details; (3) times to diagnosis and treatment; (4) treatment plan details, including enrollment on a therapeutic trial and whether the initial treatment plan was terminated early or completed as planned; and (5) outcomes such as relapse, second malignancy and death.

The reasons for non-enrollment have been consistently collected by CYP-C throughout the study period, whereas a standardised list has only recently been incorporated into POGONIS. Thus, description of reasons for non-enrollment was restricted to the 12 non-Ontario sites.

The CYP-C program achieves high-quality data through multiple approaches. A community of practice composed of each site's data manager was established to maximise the data quality through monthly teleconference and annual face-to-face training combined with site audits. The data were provided for the purpose of this analysis on 19 September 2016.

Statistical plan. Event-free survival was defined as time from diagnosis to relapse or death from any cause, whichever occurred first. Those without an event were censored on the date of last followup. Overall survival was defined as time from diagnosis to death from any cause or date of last follow-up. Survival was described for those enrolled and not enrolled on a therapeutic trial at diagnosis using the Kaplan-Meier method and compared using the log rank test.

In order to evaluate potential confounders, Cox proportional hazards models were created. The following variables were examined: (1) demographic features: age at diagnosis (1-4, 5-9 and 10-14), sex, race, and diagnostic era $(<2007 v s \geqslant 2007$, the approximate mid- point); (2) Leukaemia features: initial white blood cell count (WBC) $\left(\geqslant 50\right.$ vs $\left.<50 \times 10^{9} 1^{-1}\right)$, central nervous system (CNS) status 1 (no blasts), 2 (presence $<5 \mu \mathrm{l}^{-1} \mathrm{WBCs}$ and cytospin positive for blasts) or 3 (CSF $>5 \mu \mathrm{l}^{-1}$ WBCs and cytospin positive for blasts), immunophenotype (B-precursor vs $\mathrm{T}$ ), and cytogenetic risk group; and (3) Socioeconomic factors: kilometers to the nearest tertiary care paediatric centre and neighborhood income quintile. We also stratified analyses by National Cancer Institute (NCI) standard (age $<10$ years and initial WBC $<50 \times 10^{9} 1^{-1}$ ) and high (age $\geqslant 10$ years or initial $\mathrm{WBC} \geqslant 50 \times 10^{9} 1^{-1}$ ) risk groups. Favourable cytogenetics were defined as trisomies 4 and 10 and $t(12 ; 21)$. Unfavourable cytogenetics were defined as hypodiploidy $(<45$ chromosomes), $t(9 ; 22)$, MLL (11q23) rearrangements, and RUNX1 (AML1) amplification.

We used postal codes at diagnosis to determine distance to the nearest tertiary care paediatric cancer centre and area-level socioeconomic status by linking to the census data. Full 6 digit postal codes were available for all provinces except for British Columbia, in which 3 digit postal codes were available. Using the Statistics Canada Postal Code Conversion File software (PCCF +, Version 4J), we linked the postal code to a 2001 census dissemination area. Dissemination areas are the smallest area unit defined by Statistics Canada and include between 400 and 700 persons. Using the 2001 census, we determined income quintiles that adjust for household size and regional differences (Borugian et al, 2005).

Adjusted associations between enrollment on trials and survival outcomes were described using hazard ratios (HR) with 95\% confidence intervals (CIs). Models adjusted for all demographic, leukaemic and socioeconomic factors separately and then together. Statistical significance was defined as $P$-value $<0.05$. Statistical analysis was conducted using the SAS statistical program (SAS-PC, version 9.4; SAS Institute Inc, Cary, NC, USA).

\section{RESULTS}

There were 2732 identified children with ALL in CYP-C, among whom 72 had unknown enrollment status, 76 were infants $<1$ year of age and 15 had Burkitt's leukaemic, leaving 2569 patients available for analysis. Of the 2569 included patients, 1408 (54.8\%) were enrolled on a clinical trial at diagnosis. For the 1408 enrolled on a clinical trial, 562 (39.9\%) were enrolled on Children's Oncology Group protocols, 336 (23.9\%) were enrolled on Dana Farber Cancer Institute ALL Consortium protocols and the remainder were others or unknown. Conversely, for the 1161 not enrolled on a trial, 506 (43.6\%) were treated according to Children's Oncology Group protocols, 78 (6.7\%) were treated according to Dana Farber Cancer Institute protocols and the remainder were others or unknown.

Table 1 shows the demographic, leukaemic and socioeconomic features of the study cohort by enrollment status. Those enrolled were more likely to be white, have an initial WBC $<50 \times 10^{9} 1^{-1}$, CNS 2 status, B precursor immunophenotype and favourable cytogenetic features. Table 2 illustrates the reasons for non-enrollments for centres outside of Ontario and shows that the most common known reason for non-enrollment was lack of an available trial.

Event-free survival at 5 years was $89.8 \% \pm 0.9$ vs $84.1 \% \pm 1.2$, $P<0.0001$, for those enrolled and not enrolled on a therapeutic trial at diagnosis respectively (Figure 1). Overall survival at 5 years was $94.1 \% \pm 0.7$ vs $90.5 \% \pm 1.0, P=0.001$ (Figure 2). Table 3 shows univariate Cox proportional hazards models evaluating factors associated with survival. Enrollment on trials was significantly associated with better EFS (HR: 0.62, 95\% CI: 0.49-0.78; $P<$ 0.0001 ) and OS (HR: 0.61, 95\% CI: $0.45-0.82 ; P=0.001)$. When stratified by NCI risk status and presented by those enrolled $v s$ not enrolled, EFS was $91.0 \pm 1.0$ vs $86.8 \pm 1.3(P=0.006)$ for 


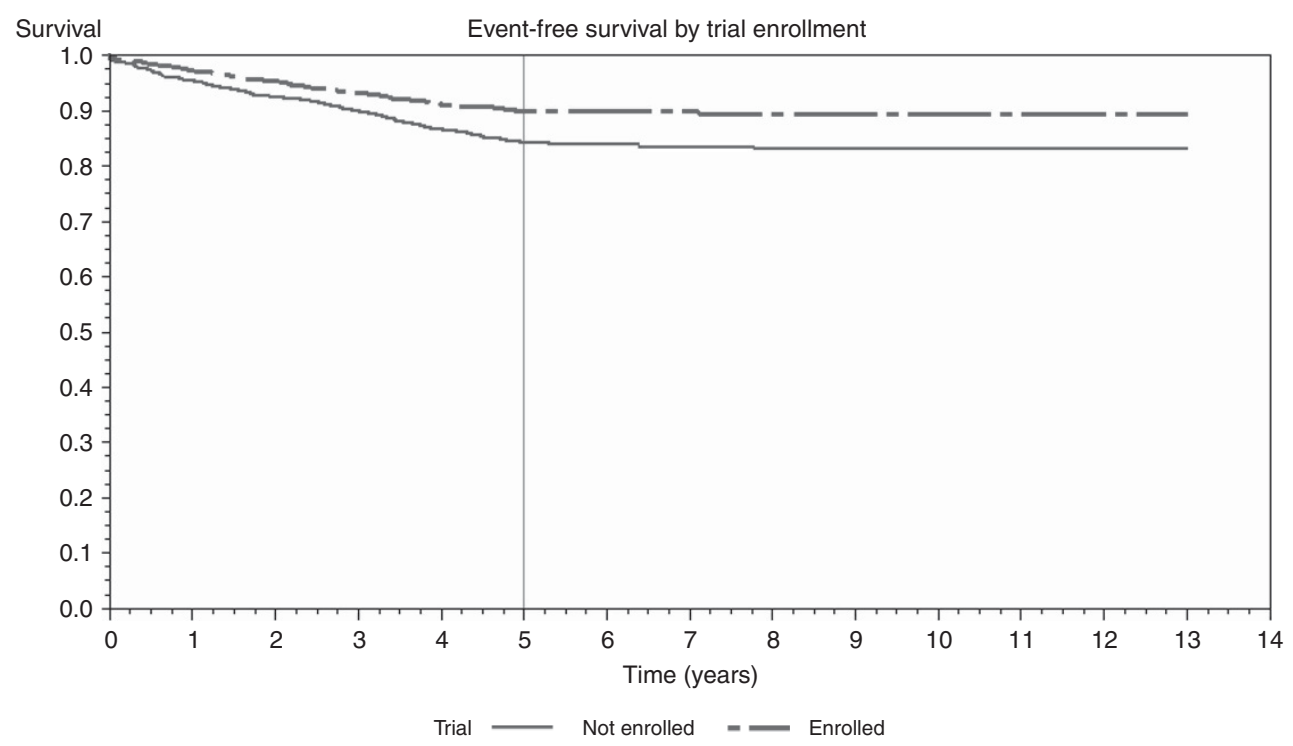

Figure 1. Event-free survival for children with acute lymphoblastic leukaemic enrolled and not enrolled on a clinical trial.

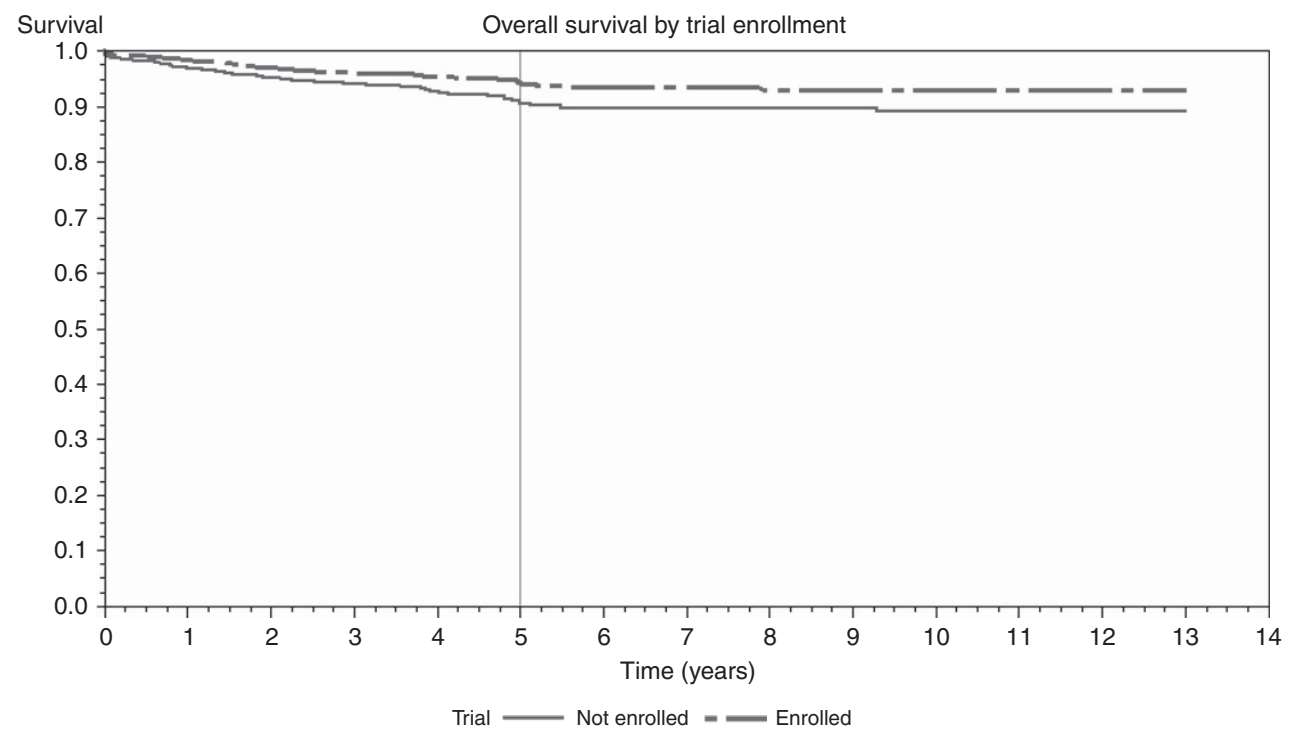

Figure 2. Overall survival for children with acute lymphoblastic leukaemic enrolled and not enrolled on a clinical trial.

standard-risk patients and was $86.9 \pm 1.8$ vs $78.7 \pm 2.3(P=0.006)$ for high-risk patients, respectively. Overall survival was $96.2 \pm 0.7$ vs $93.4 \pm 1.0(P=0.014)$ for standard-risk patients and was $88.5 \pm 1.9$ vs $84.1 \pm 2.3(P=0.093)$ for high-risk patients.

Table 4 illustrates adjusted analyses by demographic, leukaemic and socioeconomic factors and adjustment for all factors together. In the fully adjusted model, enrollment on trials was significantly associated with better EFS (HR: $0.67,95 \%$ CI: $0.47-0.95 ; P=0.023$ ) but not OS (HR: $0.69,95 \% \mathrm{CI}: 0.44-1.08 ; P=0.102$ ). To determine if the impact of enrollment on EFS differed by age group, an interaction term was evaluated in a multivariable model which included enrollment and age. The $P$-value for interaction was 0.870 suggesting a similar effect across age groups.

When examining the proportion of treatment plans terminated early rather than completed as planned, 249 out of $1408(17.7 \%)$ for those enrolled were terminated early compared to 156 out of $1161(13.4 \%)$ for those not enrolled $(P=0.004)$. When the analysis was restricted to children with ALL treated according to Children's Oncology Group trials, enrollment was associated with better EFS (HR: $0.61,95 \%$ CI: $0.42-0.88 ; P=0.008$ ) and OS (HR: $0.46,95 \%$
CI: $0.27-0.76 ; P=0.003)$. This benefit was not seen when the analysis was restricted to children treated according to Dana Farber Cancer Institute trials; enrollment was not associated with better EFS (HR: $0.75,95 \%$ CI: $0.35-1.63 ; P=0.475$ ) or OS (HR: $1.11,95 \%$ CI: $0.39-3.21 ; P=0.843)$.

\section{DISCUSSION}

We found that enrollment on a therapeutic clinical trial at initial leukaemic diagnosis was independently associated with better EFS for children newly diagnosed with ALL after adjustment for demographic, leukaemic and socioeconomic factors. Improved EFS was also seen when stratified by NCI risk status. This information may be important to families and clinicians when deciding whether to enroll on a clinical trial at diagnosis.

Our results are discordant with (Koschmann et al, 2010) who evaluated the trial effect among paediatric ALL patients treated at Seattle Children's Hospital from 1997 to 2005. They failed to demonstrate an EFS advantage to participation in studies However, 
Table 1. Demographics of acute lymphoblastic leukaemia study population by enrollment status

\begin{tabular}{|c|c|c|c|c|c|}
\hline Characteristics & $\begin{array}{c}\text { Total } \\
N=2569\end{array}$ & $\begin{array}{l}\text { Enrolled } \\
(N=1408)\end{array}$ & $\begin{array}{c}\text { Not enrolled } \\
(N=1161)\end{array}$ & $\begin{array}{l}\text { Percent } \\
\text { enrolled }\end{array}$ & $P$-value \\
\hline \multicolumn{6}{|l|}{ Demographic features } \\
\hline Age at diagnosis & & & & & 0.501 \\
\hline $5-9$ years & $732(28.5 \%)$ & $393(27.9 \%)$ & 339 (29.2\%) & 53.7 & \\
\hline $10-14$ years & $426(16.6 \%)$ & $227(16.1 \%)$ & $199(17.1 \%)$ & 53.3 & \\
\hline Male sex & $1451(56.5 \%)$ & 810 (57.5\%) & 641 (55.2\%) & 55.8 & 0.238 \\
\hline White & $1752(68.2 \%)$ & $1051(74.6 \%)$ & 701 (60.4\%) & 60.0 & \\
\hline Asian & $306(11.9 \%)$ & $132(9.4 \%)$ & 174 (15.0\%) & 43.1 & \\
\hline Arab/West Asian & $48(1.9 \%)$ & $24(1.7 \%)$ & $24(2.1 \%)$ & 50.0 & \\
\hline Aboriginal & $73(2.8 \%)$ & $39(2.8 \%)$ & $34(2.9 \%)$ & 53.4 & \\
\hline Black & $54(2.1 \%)$ & $28(2.0 \%)$ & $26(2.2 \%)$ & 51.9 & \\
\hline Latin American & 37 (1.4\%) & $17(1.2 \%)$ & $20(1.7 \%)$ & 45.9 & \\
\hline Other & $52(2.0 \%)$ & $25(1.8 \%)$ & $27(2.3 \%)$ & 48.1 & \\
\hline \multicolumn{6}{|l|}{ Leukaemia features } \\
\hline Initial white blood cell count & & & & & \\
\hline$<50 \times 10^{9} \mathrm{I}^{-1}$ & $2115(82.3 \%)$ & $1190(84.5 \%)$ & 925 (79.7\%) & 56.3 & 0.002 \\
\hline$\geqslant 50 \times 10^{9} \mathrm{I}^{-1}$ & $454(17.7 \%)$ & $218(15.5 \%)$ & 236 (20.3\%) & 48.0 & \\
\hline \multicolumn{6}{|l|}{ CNS status } \\
\hline CNS1 & $2292(89.2 \%)$ & $1237(87.9 \%)$ & 1055 (90.9\%) & 54.0 & 0.002 \\
\hline CNS2 & $217(8.4 \%)$ & $142(10.1 \%)$ & 75 (6.5\%) & 65.4 & \\
\hline CNS3 & $53(2.1 \%)$ & $28(2.0 \%)$ & $25(2.2 \%)$ & 52.8 & \\
\hline Unknown & 7 (0.3\%) & 1 (0.1\%) & $6(0.5 \%)$ & 14.3 & \\
\hline \multicolumn{5}{|l|}{ Immunophenotype } & 0.0006 \\
\hline B-precursor & $1313(51.1 \%)$ & $810(57.5 \%)$ & 503 (43.3\%) & 61.7 & \\
\hline $\mathrm{T}$ & $159(6.2 \%)$ & $75(5.3 \%)$ & $84(7.2 \%)$ & 47.2 & \\
\hline \multicolumn{5}{|l|}{ Income quintile } & 0.485 \\
\hline 1 (lowest) & $486(18.9 \%)$ & 254 (18.0\%) & $232(20.0 \%)$ & 52.3 & \\
\hline 2 & 479 (18.6\%) & 259 (18.4\%) & 220 (18.9\%) & 54.1 & \\
\hline 3 & 517 (20.1\%) & $298(21.2 \%)$ & $219(18.9 \%)$ & 57.6 & \\
\hline 4 & 537 (20.9\%) & 292 (20.7\%) & 245 (21.1\%) & 54.4 & \\
\hline 5 (highest) & 502 (19.5\%) & $282(20.0 \%)$ & 220 (18.9\%) & 56.2 & \\
\hline Missing & 48 & 23 & 25 & & \\
\hline
\end{tabular}

Table 2. Reasons for non-enrollment on trials in 12 nonOntario institutions

\begin{tabular}{|l|c|}
\hline & Total $(\mathbf{N}=\mathbf{5 9 5})$ \\
\hline No available trial $^{\text {a }}$ & $319(53.6 \%)$ \\
\hline Language barrier, trial not offered $^{\text {Not eligible for any trial }}{ }^{\text {a }}$ & $9(1.5 \%)$ \\
\hline Physician choice & $24(9.1 \%)$ \\
\hline Refused therapy & $5(0.8 \%)$ \\
\hline Refused to participate in trial & $62(10.4 \%)$ \\
\hline Other & $14(2.4 \%)$ \\
\hline $\begin{array}{l}\text { Unknown } \\
\text { a No available trial suggests that a trial was not available for a specific disease. In contrast, } \\
\text { not eligible for any trial suggests that a trial was available for a patient's disease but the } \\
\text { patient did not meet that trial's eligibility criteria. }\end{array}$ \\
\hline
\end{tabular}

this report was limited since it included only a single centre in the United States. Further, the sample size consisted of only 322 patients (with $48.8 \%$ enrolment).

Reasons why enrollment on trials could improve EFS include the following: (1) Treatment effect, in which the interventions being evaluated result in better outcomes compared with standard approaches; (2) Participation effect where enrollment in the trial results in better outcomes due to the effect of the protocol, changes in healthcare professional behavior, changes in patient/family behavior or a placebo effect; and (3) Confounding, if patients enrolled on trials are systematically different than patients not enrolled on trials. The adjusted analyses were important as those enrolled on trials, when compared to those not enrolled, had generally favourable features such as low initial WBC, B precursor immunophenotype and favourable risk cytogenetics. By taking into consideration confounding using several approaches, our study suggests that improved EFS may be the result of the interventions being evaluated in these studies and is 
Table 3. Impact of trial enrollment on event-free and overall survival ${ }^{\mathrm{a}}$

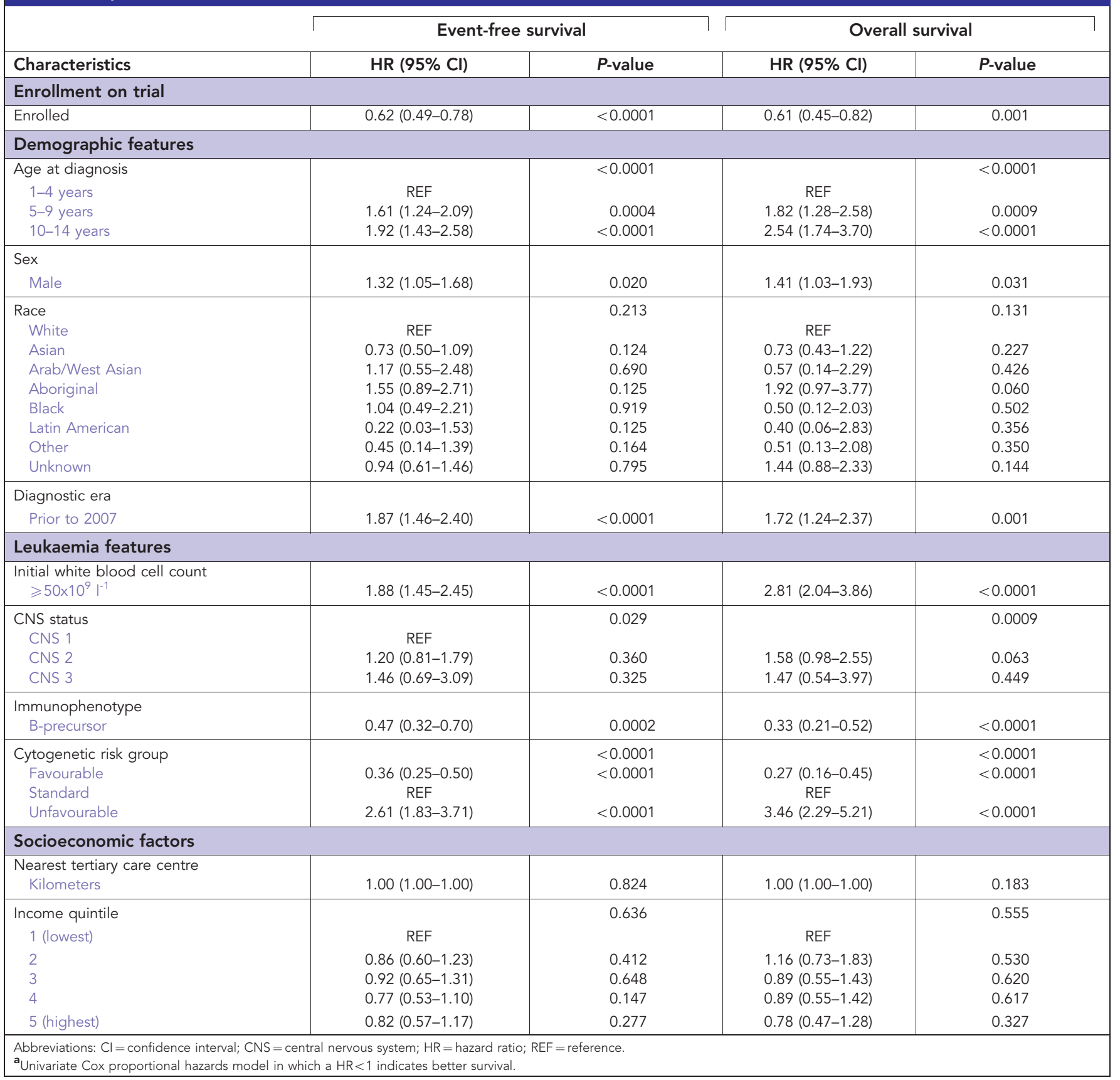

consistent with improved outcomes in paediatric ALL with successive clinical trials (Pui et al, 2015). However, we cannot exclude that participation effect may have a role as well. If improved EFS is the result of interventions being evaluated, this finding may not apply to trials examining de-escalation of therapy.

We also found that when patients were treated with COG protocols, patients enrolled had better outcomes compared to those not enrolled, whereas a similar pattern was not seen among those treated according to Dana Farber Cancer Institute protocols. This finding may be related to either the interventions being evaluated in specific trials or may be related to the smaller number of children receiving Dana Farber Cancer Institute studies. We also found that those enrolled on trials were more likely to terminate the treatment plan early compared to those not enrolled on trials. This finding may be an artefact of how the data were collected since if patients enrolled on trials were taken off protocol therapy but continued to follow the same treatment protocol, they were designated as terminating treatment early and starting a new treatment plan as a non-registered patient.

Our results were less definitive regarding whether enrollment on trials improves OS and such an effect is difficult to demonstrate in a disease with high success rates in general. While we found that OS was not improved in the adjusted models, it should be noted that the HR favoured enrollment and that power was limited given excellent survival. Thus, combining the CYP-C data set with other populationbased registries may help us understand whether enrollment on trials can reduce mortality.

The strengths of this study are its population-based nature and careful collection of confounders, including leukaemic and socioeconomic factors. Other strengths are the high quality of data and common health care system, which provides universal healthcare. However, these results must be interpreted in light of its limitations. First, potentially important covariates were not available such as minimal residual disease (MRD) status. Second, 
Table 4. Impact of enrollment on trials on event-free and overall survival in adjusted models ${ }^{a}$

\begin{tabular}{|c|c|c|c|c|}
\hline \multirow[b]{2}{*}{ Characteristics } & \multicolumn{2}{|c|}{ Event-free survival } & \multicolumn{2}{|c|}{ Overall survival } \\
\hline & $\mathrm{HR}(95 \% \mathrm{Cl})$ & $P$-value & $\mathrm{HR}(95 \% \mathrm{Cl})$ & $P$-value \\
\hline \multicolumn{5}{|l|}{ Variables included in multiple regression } \\
\hline Demographic: age, gender, ethnicity, era & $0.60(0.48-0.76)$ & $<0.0001$ & $0.61(0.45-0.82)$ & 0.001 \\
\hline $\begin{array}{l}\text { Leukaemia: white count, CNS status, immunophenotype, } \\
\text { cytogenetics }\end{array}$ & $0.72(0.53-0.99)$ & 0.040 & $0.75(0.50-1.13)$ & 0.163 \\
\hline Socioeconomic: distance and income quintile & $0.64(0.50-0.82)$ & 0.0003 & $0.63(0.45-0.86)$ & 0.004 \\
\hline Demographic, leukaemic and socioeconomic factors & $0.67(0.47-0.95)$ & 0.023 & $0.69(0.44-1.08)$ & 0.102 \\
\hline
\end{tabular}

we did not include adolescent and young adult patients (AYA) in our study. This is important as several studies have identified that AYA patients have lower rates of enrollment on clinical trials (Bleyer, 2002; Downs-Canner and Shaw, 2009; Aristizabal et al, 2015). Third, we lacked immunophenotype information for over $40 \%$ of children related to how POGO classifies ALL; this missing data affected the power of analyses, which adjusted for leukaemicrelated factors. Finally, we used a census based measure of family income as individually reported data from the family were not available at the population level.

In conclusion, EFS was significantly better in children with ALL enrolled on a clinical trial. Future research should identify barriers to clinical trial enrollment for children with ALL.

\section{ACKNOWLEDGEMENTS}

The authors gratefully acknowledge the contributions of study participants, participating paediatric oncology centres, members of the Cancer in Young People in Canada (CYP-C) Management and Steering Committees, the Paediatric Oncology Group of Ontario (POGO) and the five POGO Hospital Partners. The CYP-C is fully funded by the Public Health Agency of Canada. We wish to thank all the data managers at the 17 CYP-C sites for their dedicated work in maintaining the CYP-C data quality and Dr. Mark Bernstein for his leadership in CYP-C development. We also thank Jay Onysko for his management of the CYP-C program and constructive comments on a draft version of this manuscript.

\section{CONFLICT OF INTEREST}

The authors declare no conflict of interest.

\section{ETHICAL APPROVAL}

The study received Research Ethics Board approval from the coordinating site (The Hospital for Sick Children). For sites outside of Ontario, Research Ethics Board approval was obtained for collection of CYP-C data. For the five Ontario sites, the requirement for Research Ethics Board approval was waived given the Paediatric Oncology Group of Ontario's status as a prescribed 45 Entity under the Personal Health Information Protection Act, 2004. This status allows the Paediatric Oncology Group of Ontario to be a custodian of personal health information for the province. The requirement for informed consent and assent were waived given the retrospective nature of the study.

\section{REFERENCES}

Aristizabal P, Singer J, Cooper R, Wells KJ, Nodora J, Milburn M, Gahagan S, Schiff DE, Martinez ME (2015) Participation in paediatric oncology research protocols: Racial/ethnic, language and age-based disparities. Pediatr Blood Cancer 62: 1337-1344.

Bleyer WA (2002) Cancer in older adolescents and young adults: epidemiology, diagnosis, treatment, survival, and importance of clinical trials. Med Pediatr Oncol 38: 1-10.

Borugian MJ, Spinelli JJ, Mezei G, Wilkins R, Abanto Z, Mcbride ML (2005) Childhood leukaemic and socioeconomic status in Canada. Epidemiology 16: 526-531.

Downs-Canner S, Shaw PH (2009) A comparison of clinical trial enrollment between adolescent and young adult (AYA) oncology patients treated at affiliated adult and paediatric oncology centres. J Pediatr Hematol Oncol 31: 927-929.

Koschmann C, Thomson B, Hawkins DS (2010) No evidence of a trial effect in newly diagnosed paediatric acute lymphoblastic leukaemic. Arch Pediatr Adolesc Med 164: 214-217.

Peppercorn JM, Weeks JC, Cook EF, Joffe S (2004) Comparison of outcomes in cancer patients treated within and outside clinical trials: conceptual framework and structured review. Lancet 363: 263-270.

Pole JD, Barber R, Bergeron RE, Carret AS, Dix D, Kulkarni K, Martineau E, Randall A, Stammers D, Strahlendorf C, Strother DR, Truong TH, Sung L (2017) Most children with cancer are not enrolled on a clinical trial in Canada: a population-based study. BMC Cancer 17: 402.

Pui CH, Yang JJ, Hunger SP, Pieters R, Schrappe M, Biondi A, Vora A, Baruchel A, Silverman LB, Schmiegelow K, Escherich G, Horibe K, Benoit YC, Izraeli S, Yeoh AE, Liang DC, Downing JR, Evans WE, Relling MV, Mullighan CG (2015) Childhood acute

lymphoblastic leukaemic: progress through collaboration. J Clin Oncol 33: 2938-2948.

Stiller CA, Eatock EM (1994) Survival from acute non-lymphocytic leukaemia, 1971-88: a population based study. Arch Dis Child 70: 219-223.

Vist GE, Bryant D, Somerville L, Birminghem T, Oxman AD (2008) Outcomes of patients who participate in randomized controlled trials compared to similar patients receiving similar interventions who do not participate. Cochrane Database Syst Rev MR000009, Issue 3.

Wagner HP, Dingeldein-Bettler I, Berchthold W, Luthy AR, Hirt A, Pluss HJ, Beck D, Wyss M, Signer E, Imbach P et al. (1995) Childhood NHL in Switzerland: incidence and survival of 120 study and 42 non-study patients. Med Pediatr Oncol 24: 281-286.

This work is published under the standard license to publish agreement. After 12 months the work will become freely available and the license terms will switch to a Creative Commons AttributionNonCommercial-Share Alike 4.0 Unported License. 